\title{
Optical layout of the fluorescence detector of the Pierre Auger Observatory
}

\author{
Miroslav Palatka', Petr Schovanek', Miroslav Hrabovsky', Martin Vlcek', Jan Ridky ${ }^{2}$, \\ Jiri Grygar ${ }^{2}$, Ladislav Soukup ${ }^{2}$, Michael Prouza ${ }^{2}$, Martina Bohacova ${ }^{2}$ \\ 'Joint Laboratory of Optics of Palacky University and Institute of Physics of the Academy of Sciences \\ of the Czech Republic, 17 Listopadu 50, 77207 Olomouc, Czech Republic, e-mail: palatka@sloup.upol.cz. \\ ${ }^{2}$ Institute of Physics of the Academy of Sciences of the Czech Republic, 17 Listopadu 50, 77207 Olomouc, \\ Czech Republic.
}

We describe an unusual optical layout of the fluorescence detector telescope which is a part of the Pierre Auger Observatory. The fluorescence detector is a very fast telescope with the relative aperture $f_{\text {no }} \sim 0.77$ and the large field of view $30 \times 30$ degrees. The primary objective for the Auger fluorescence telescope is to measure accurately the longitudinal development profiles of air showers of the cosmic rays above $10 \mathrm{EeV}$ energy.

Keywords: fluorescence detector, optical layout.

\section{Introduction}

The origin of the highest energy cosmic rays is still not known and is the burning question in high-energy astrophysics research. Unfortunately, very high energy cosmic rays are very rare (at $10^{20} \mathrm{eV}$ only 1 cosmic ray hits each square kilometer of the Earth's surface per century) and so a huge detector must be built in order to detect them. The proposed Pierre Auger Observatory would be just such a detector. The Pierre Auger Observatory will eventually consist of two observatories, one in the northern hemisphere and one in the south. Each will have 1600 Cherenkov water detectors spread over $3000 \mathrm{~km}^{2}$ and 4 fluorescence light detectors. The first part of the world biggest cosmic ray observatory is being built in Argentina's Mendoza province and will be launched in 2004 (Fig. 1). Its telescopes will scour the heavens for clues, which may solve mysteries surrounding the Big Bang, black holes and sub-atomic particles.

\section{Principle of operation}

Cosmic rays are fast-moving particles from space that constantly bombard the earth from all directions. Each second, about 200 cosmic ray particles with energies of a few million electron volts $\left(10^{6} \mathrm{eV}\right)$ strike every square meter of the earth. While these low 

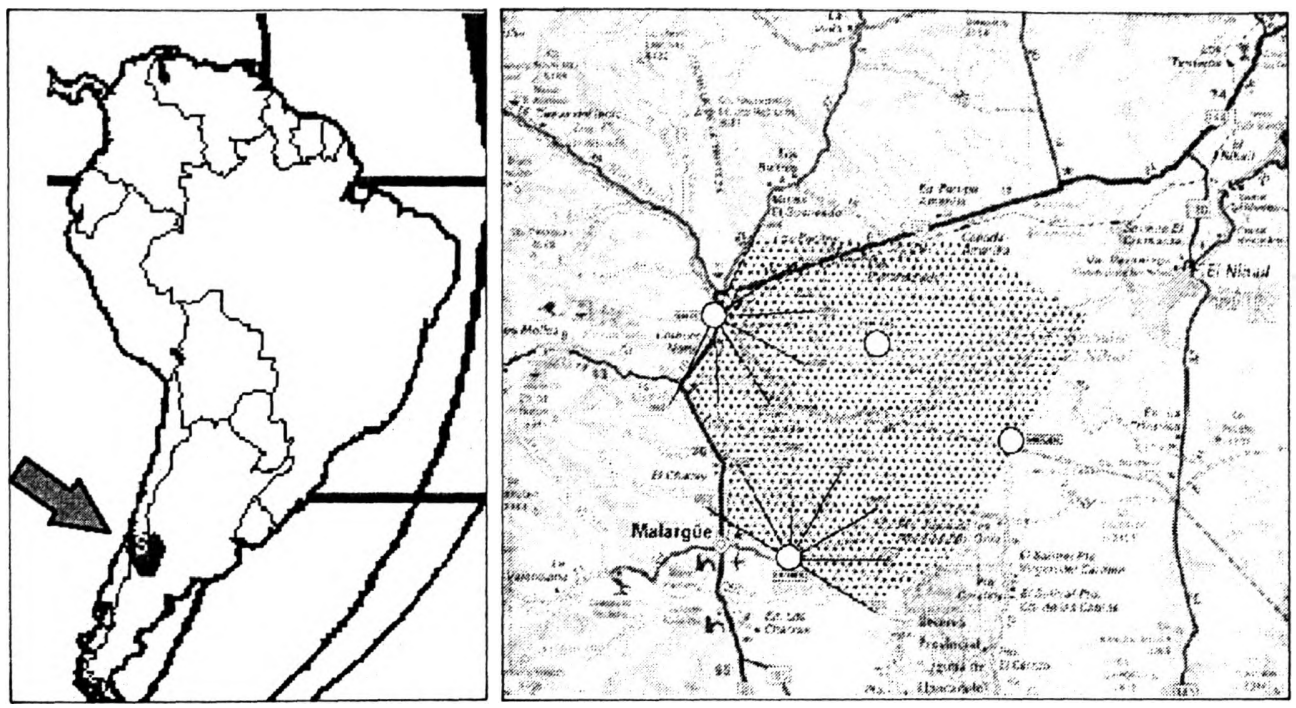

Fig. 1. Array of Cherenkov water detectors and four FD positions planned.

-energy cosmic rays are plentiful, cosmic rays at higher energies are far rarer. Above the energy of $10^{18} \mathrm{eV}$, only one particle falls on an area of one square kilometer each week. Above the energy of $10^{20} \mathrm{eV}$, only one particle falls on one square kilometer in a century. To find and measure these rare events, a high-energy cosmic ray study needs to wait centuries or to build a truly giant detector.

To measure cosmic ray particles directly requires sending detectors to heights above most of the earth's atmosphere, using high-flying balloons and satellites. However, we can also detect cosmic rays indirectly on the surface of the earth by observing the showers of particles that they produce in the air. Extensive air showers are large cascades of electrons, photons, muons and other sub-nuclear particles that are produced when high energy protons or heavy nuclei from deep space collide with the Earth's upper atmosphere. The myriads of secondary particles, which are produced during the development of these extensive air showers, are registered with special detectors on the ground. The array of these detectors consists of 1600 water tanks arranged in grid with spacing of $1.5 \mathrm{~km}$ and covering about $3000 \mathrm{~km}^{2}$. The tanks are filled with purified water and large photomultiplier tubes will view the water volume vertically downwards from above. Upon entering the water all air shower particles produce Cherenkov light which is then detected by the photomultipliers.

This method only sees particles that reach the ground, but do not tell us about how a cosmic ray shower develops in the atmosphere. A new technique was developed in the 1980s based on the phenomenon of the atmospheric fluorescence. When a charged particle passes close to molecules in the atmosphere, it transfers some energy to the molecules, in effect "shaking up" the electrons inside. The molecules respond by emitting light as their electrons return to their normal arrangement, and this effect is known as fluorescence radiation. Nitrogen molecules, which make up most of the air, 


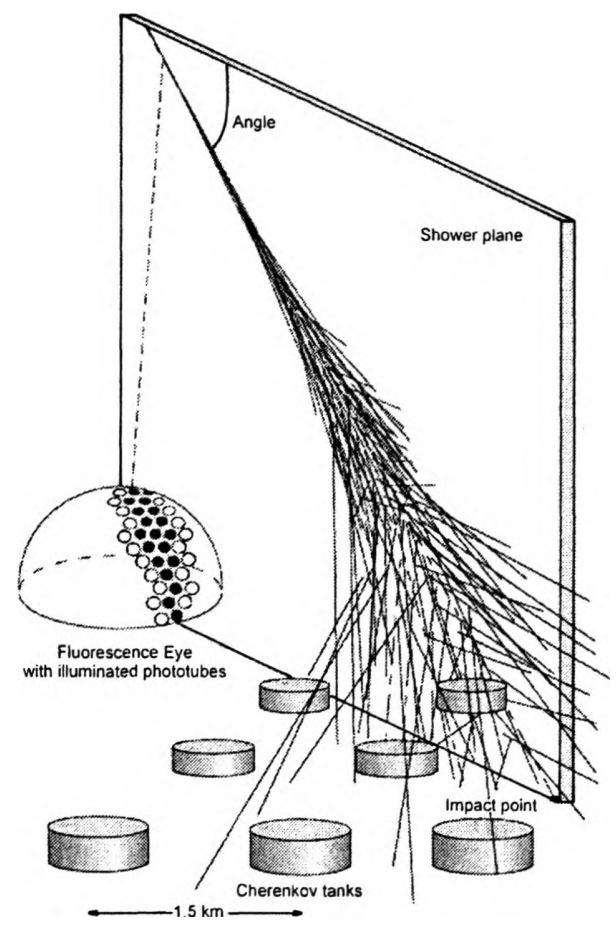

Fig. 2. Illustration of the operation of air shower detectors.

emit blue fluorescent light. Sensitive UV detectors (photo multipliers) at a focal surface of special telescope can see this light. This technique was first successfully implemented twenty years ago by the University of Utah with their "Fly's Eye" detector and is illustrated together with the previous method in Fig. 2. The primary role of the fluorescence method is to measure the longitudinal profile of each shower development in atmosphere.

\section{Fluorescence detector layout}

The fluorescence detector (FD) observes cosmic rays in much the same way as a video camera might observe meteor trails. The difference is that the air shower flash is much fainter and has a much greater angular velocity. The FD will consist on four "eyes" which overlook the array of Cherenkov water detectors (Fig. 2). The three of them will be near the perimeter of the surface array and the last roughly in the central part at locations which are slightly elevated with respect to the rest of the detector. The perimeter eyes consist of 6 telescopes (Fig. 3) and the central eye consists of 12 telescopes.

The proposal provides the central eye with a $360^{\circ}$ and the perimeter eye with a $180^{\circ}$ field of view in azimuth. Each eye views $30^{\circ}$ upwards from the horizon so each telescope has $30 \times 30^{\circ}$ field of view. The design of the telescope calls for a wide angle and fast 


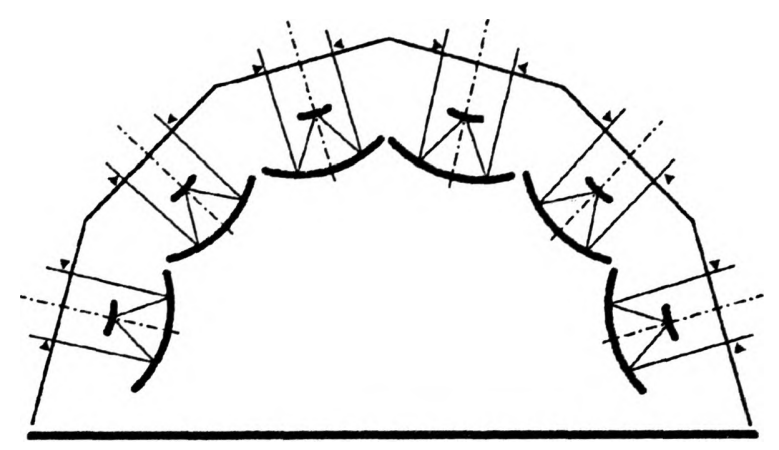

Fig. 3. Schematic illustration of the perimeter eye.

optical system. This telescope will collect the slight fluorescence light of air shower onto camera with photomultipliers. The desired resolution on the measurement of the shower profile implies the pixel size not larger than $1.5^{\circ}$ and the sensitivity of detector requires an aperture with at least $1.5 \mathrm{~m}^{2}$ effective area for the light collection [1].

The lensless Schmidt camera design was adopted as a good solution of fast and wide-angle telescope systems. As a reasonable compromise between the optical quality and the cost was the choice of the camera with relative aperture $f / 1$. The required light collection area leads to $1700 \mathrm{~mm}$ diameter of the diaphragm when the obscuration of PMT camera is taken into account. The radius of the mirror is then $3400 \mathrm{~mm}$ and the angular size of the spot given by spherical aberration comes out to be $0.5^{\circ}$. It is $1 / 3$ of the pixel size. The mirror size is nearly square due to the square field of view with dimension ca $3500 \times 3500 \mathrm{~mm}$.

\section{Final fluorescence telescope design - unusual optical layout}

\subsection{Optical properties of lensless Schmidt camera - overview}

The lensless Schmidt camera is the fundamental form of a wide field telescope without a correction of the spherical aberration. It consists of a stop at the centre of curvature of a spherical mirror. The system has only two aberrations, field curvature and the spherical aberration, which is identical for all field directions apart from stop vignetting. The focal "plane" is concentric with a mirror and its radius of curvature is equal to the paraxial focal length. The rays near the paraxial focus point touch a surface of revolution called the caustic. The smallest image is where the ray from the rim of the pupil meets the caustic. The smallest image patch is called the disc of least confusion. The best detector place is therefore shifted from paraxial focus and the radius of detector surface is changed.

The lensless Schmidt fluorescence telescope has the following optical parameters [1], [2]:

- focal length $f=1700 \mathrm{~mm}$,

- aperture of the diaphragm $D=1700 \mathrm{~mm}$,

- field of view $30 \times 30^{\circ}$ (a half-field of view is $20^{\circ}$ due to square camera detector), 

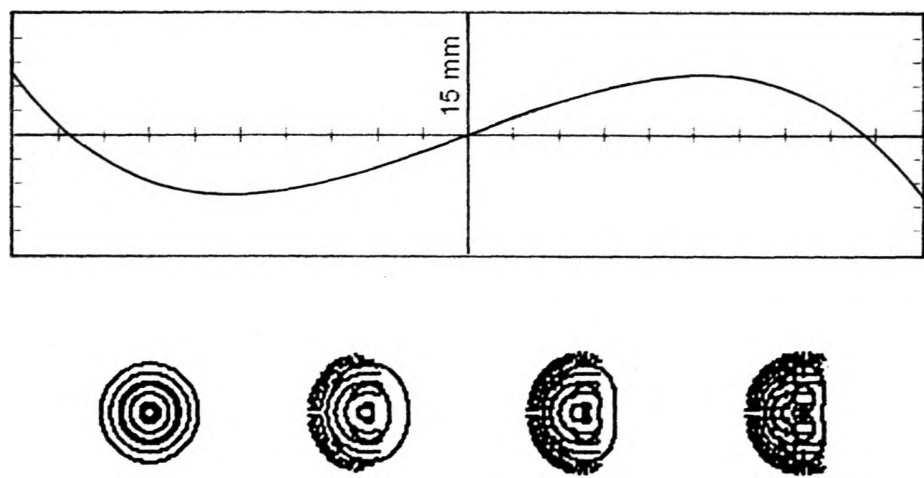

b

Fig. 4. Lensless Schmidt camera: a - transverse aberration (the same for $0-20^{\circ}$ fields of view), b-spot diagrams for $0^{\circ}, 10^{\circ}, 15^{\circ}, 20^{\circ}$ fields of view (from the left) .

- radius of curvature of the mirrors $R=3400 \mathrm{~mm}$,

- dimension of the mirrors $3500 \times 3500 \mathrm{~mm}$,

- radius of curvature of the detectors $R_{\mathrm{c}}=1742 \mathrm{~mm}$.

The diameter of the disc of least confusion is $d=14.83 \mathrm{~mm}$ (the transverse spherical aberration).

Figure 4 shows the aberrations of the lensless Schmidt camera. There is seen a detector obscuration for larger fields of view. The focal surface is shifted to the place of the disc of least confusion.

\subsection{Optical properties of an ordinary Schmidt camera - overview}

The ordinary Schmidt camera has an aspheric corrector plate at the center of curvature of the mirror (aperture stop). The spherical aberration is corrected without introducing a coma and an astigmatism of the third order. The aspheric corrector is a refracting element and it corrects the spherical aberration at the nominal wavelength only. At shorter or longer wavelengths the spherical aberration is overcorrected or undercorrected, respectively. This is an ordinary spherochromatism, which can be balanced by introducing a small amount of a longitudinal spherical aberration using some weak positive power to the axis of the corrector plate. A central part of the corrector is then convex and the margin of the corrector is concave. The profile shape of aspheric then satisfies the following sixth order equation:

$$
Z=A y^{2}+B y^{4}+C y^{6}
$$

where $Z$ is the depth of the curve, $y$ is the off-axis distance, and $A, B, C$ are constants depending on the refractive index of the material, the focal ratio, the aperture and the position of the neutral zone.

The Schmidt system with corrector plate has only one optical axis defined by an axis of symmetry of the corrector profile. This leads to asymmetries in the angles of incidence of oblique rays on the aspheric surface at points above and under the optical axis. This leads to higher order field aberrations. The oblique spherical aberration is 


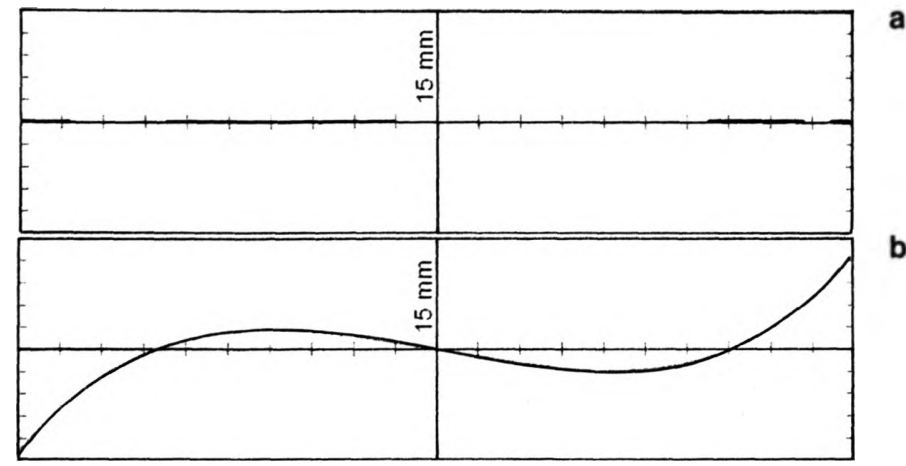

a

b

C

Fig. 5. Ordinary Schmidt camera: a - the transverse aberration for $0^{\circ}$ field of view, $\mathbf{b}-$ the transverse aberration for $20^{\circ}$ field of view, c - spot diagrams for $0^{\circ}, 10^{\circ}, 15^{\circ}, 20^{\circ}$ fields of view (from the left).

the limiting field aberration especially for fast systems as FD. Figure 5 shows the transverse aberrations and spot diagrams for example defined in Sec. 4.1.

Several participants of Pierre Auger Project studied the usefulness of corrector plate to improve the FD properties regardless of the prototype component production. The solution without any requirements on current FD parameters was fundamental condition.

\subsection{Improvement of FD optical system}

The corrector plate could be used to reduce the spot size but it can improve the FD in another way. The diameter of the FD diaphragm is determined by the required light collecting area. The given design has $1.54 \mathrm{~m}^{3}$ of the unobscured collecting area. Then the corrector could be used to enlarge the aperture (the light collecting area) without enlarging the spot size and with the same parameters of the mirror and camera.

The FD optical system was analyzed with the aim to improve the speed of existing setup based on the lensless Schmidt camera. The goal was to design a suitable optical element in the shape of an annulus. This approach has been discussed several times within the FD task group. The inclusion of the corrector ring must not deteriorate the properties of the whole optical system. The main criterion is a preservation of the maximum spot size in the picture "plane". The spot has to be contained within diameter $d=15 \mathrm{~mm}\left(0.5^{\circ}\right.$ in angular measure $)$. The construction parameters of the current optical system were already fixed and it was impossible to use them for the sake of aberration corrections. Their values are as follows [2]:

- the radius of the mirror curvature $R=3400 \mathrm{~mm}$,

- the radius of the detector curvature $r=1743 \mathrm{~mm}$,

- the distance between the mirror and the detector $s=1657 \mathrm{~mm}$,

- the diaphragm position is fixed in the plane of the center of the mirror curvature. 
Thus trying to correct the aberration of improved FD Schmidt system we are basically limited to shaping the two surfaces of the correction annulus. This annulus will have the outer diameter about $2000 \mathrm{~mm}$ and it is clear that the use of an aspheric surface is a necessity. Due to the need of the simplest manufacture of this large optical element we decided to shape only the first surface (the aspheric one) and to keep the other planar.

The main aberration of the wide field and fast ordinary Schmidt camera is a tangential oblique spherical aberration as is seen in Fig. 5. The spherical aberration is fully corrected for the field of view $0^{\circ}$ and chromatic variation of the spherical aberration is balanced. The spherochromatism is small in the case of FD in comparison with high order aberrations and it is not shown in Fig. 5 for clarity.

The amount of the oblique aberration varies as the square of the field and it is different in the tangential and sagittal directions. The spot "diameter" increases rapidly with the field of view between $0-20^{\circ}$. The goal is to get approximately the same spot "diameter" for the whole range of the field. The way for it is especially balancing of the oblique spherical aberration. This can be done with the ordinary Schmidt system by [3]: i) shifting the corrector away from the center of mirrors curvature (introduction of the primary astigmatism); ii) slightly changing the detector focal curvature ("defocusing" for larger field of view); iii) soft figuring of the main mirror (add an aspheric); iv) soft figuring of corrector plate (introduction of primary spherical aberration on axis). The fixed parameters of current FD do not allow using methods i-iii.

We introduced some spherical aberration on axis (field of view $0^{\circ}$ ) by shaping the aspheric corrector profile which is given by Eq. (1) for the FD with increasing its outer diameter above $2000 \mathrm{~mm}$. Introduction of primary spherical aberration on axis leads to reduction of transverse aberration in the case of oblique field of view. The required maximal field of view is $20^{\circ}$. The transverse aberrations are then approximately the same for field of view between $0^{\circ}$ and $20^{\circ}$ and their values must be less than $15 \mathrm{~mm}$ (original lensless spherical aberration of FD with aperture diameter $1700 \mathrm{~mm}$ ). The

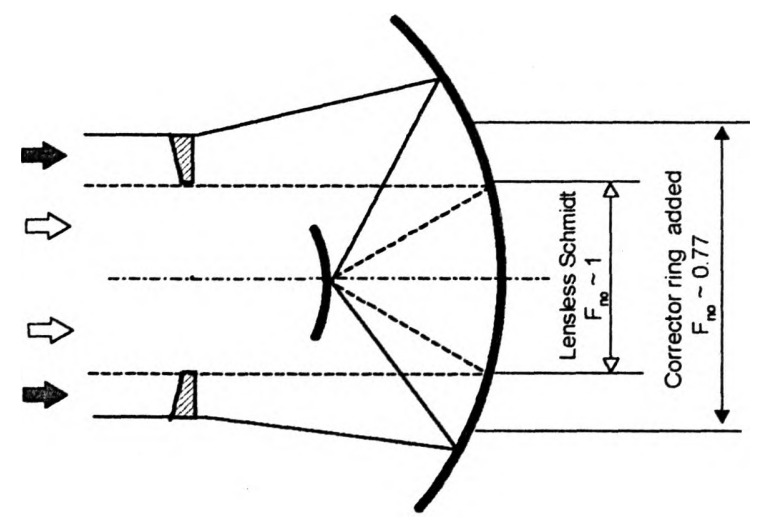

Fig. 6. Upgrade of FD optical system - illustration. 
oblique spherical aberration depends on the diameter of FD entrance aperture (the corrector lens diameter). Of course, there is some limit of this maximal diameter when the transverse aberration is balanced in required range $0-20^{\circ}$ field of view and is near the value of $15 \mathrm{~mm}$. The maximal entrance diameter found was $2200 \mathrm{~mm}$. An additional increase of the FD diameter would be possible only in the case of lesser value of maximal field of view (under $20^{\circ}$ ). The diameter of $2200 \mathrm{~mm}$ increases the focal ratio from $f / 1$ to ca $/ 0.77$ and the light collecting area increases by about $100 \%$. A schematic illustration is shown in Fig. 6.

\section{Possible profiles of a corrector optical element}

Variables of the fourth and sixth order coefficients as well as the base curvature of the aspheric surface (Eq. (1)) were used for balancing of the oblique spherical aberration. The final profile is given by the sixth order equation [4] again where the coefficients $A, B, C$ have new values. The aspheric optical element is used only in outer zone of FD aperture and it has an annular shape. It is therefore called "corrector ring" in FD task group. If we look more closely at FD optical system (Fig. 6) it is clear that we are dealing with a bifocal system composed of two subsystems. The first system is lensless Schmidt (inner part) and the second is modified ordinary Schmidt system (outer part). Figure 7 shows the transverse aberrations and spot diagrams of this optical system.
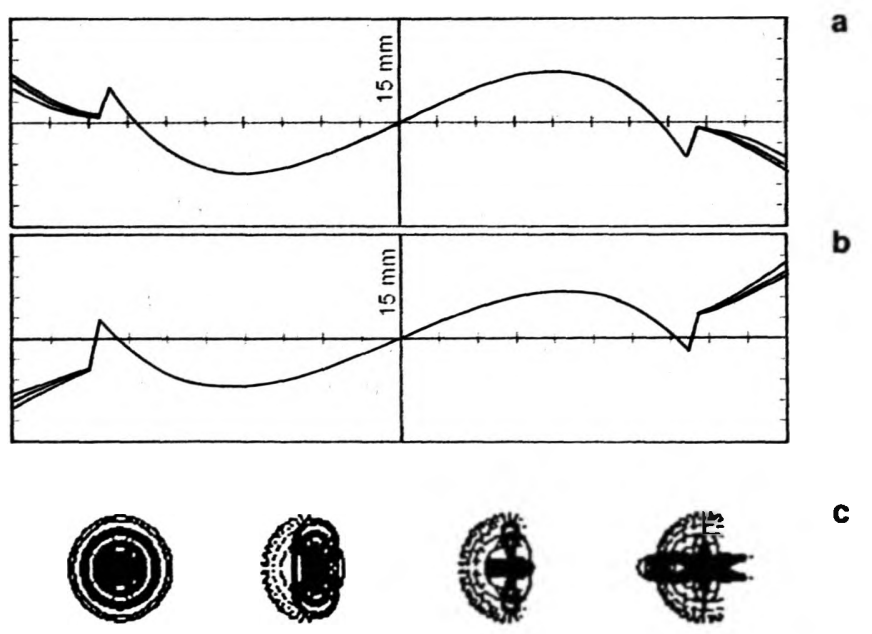

C

Fig. 7. Upgrade FD system: a - transverse aberration for $0^{\circ}$ field of view, $b$ - transverse aberration for $20^{\circ}$ field of view, $\mathrm{c}$ - spot diagrams for $0^{\circ}, 10^{\circ}, 15^{\circ}, 20^{\circ}$ fields of view (from the left).

The spot size is about $15 \mathrm{~mm}$ for all fields of view $0-20^{\circ}$ and it is ca $1 / 3$ of the required maximum value of the "pixel" size. The maximum diameter of the spot size is mainly given by outer part of the aperture (corrector ring). The role of the sixth order term in Eq. (1) is significant. We tried to remove the first two terms from this sixth order Eq. (1). We found out that it is possible to determine the corrector profile by one term polynomial only and it is applicable to the design of FD (Eq. (3)). 


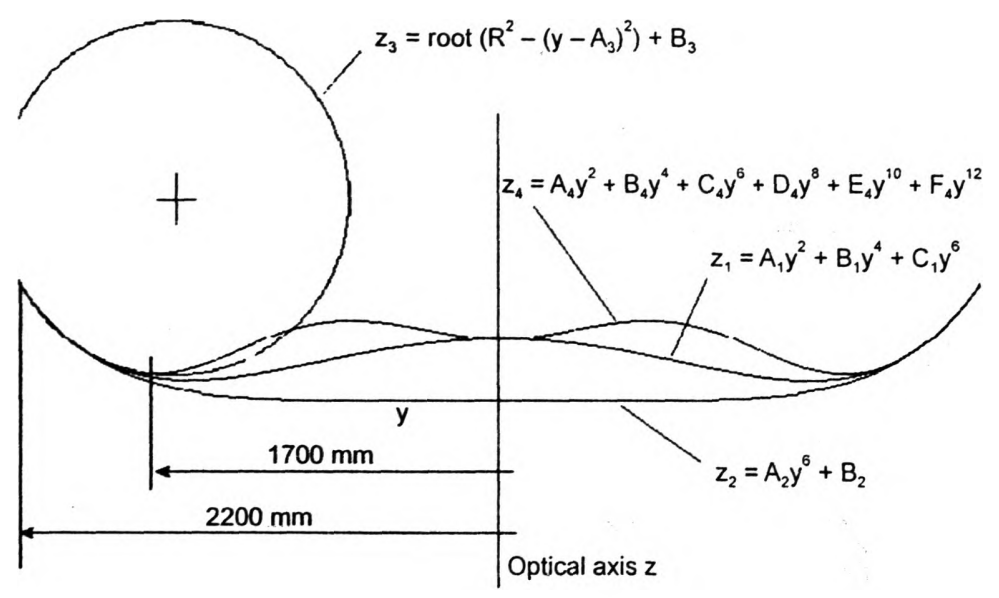

Fig. 8. Group of acceptable corrector ring profiles.

Finally, we analyzed the possibility of substituting of the aspheric surface of the corrector annulus by the shape with simpler production technology [5]. We approximated the aspheric profile by a circular shape.

Figure 8 shows a group of the corrector ring profiles which are acceptable in FD design. These are:

$$
\begin{aligned}
& z_{1}=A_{1} y^{2}+B_{1} y^{4}+C_{1} y^{6}, \\
& z_{2}=A_{2} y^{6}+B_{2}, \\
& z_{3}=\operatorname{root}\left(R_{2}-\left(y-A_{3}\right)^{2}\right)+B_{3}, \\
& z_{4}=A_{4} y^{2}+B_{4} y^{4}+C_{4} y^{6}+D_{4} y^{8}+E_{4} y^{10}+F_{4} y^{12} .
\end{aligned}
$$

The profile $z_{1}$ is a modified ordinary Schmidt plate, the profile $z_{2}$ is a new type of the shape which increases in thickness monotonically to the edge. The profile $z_{3}$ is a circular arc approximation (in the meridian plane) of $z_{1}$ profile in the outer region. This shape is suitable for manufacturing. In fact, it is a toroidal surface as a part of the special torus. The torus is usually determined by its major (inner) and minor (tube) radii. The inner radius is bigger than tube radius in our case. The profile $z_{4}$ is the 12 th order polynomial replacement of profile $z_{3}$ and was used for optical calculation instead of $z_{3}$ because this profile is impossible to add to an optical analysis program [6]. The profile $z_{3}$ of the corrector ring was chosen for manufacturing the final version of FD. The aberrations and spots of the design are seen in Fig. 9.

Of course, the substitution of the aspheric profile (Eq. (2)) by "circular arc" blurs the origin spot size. It is seen especially in the case of $0^{\circ}$ field of view (compare Figs. 7 and 9). The spot size for $0^{\circ}$ field of view is slightly larger than required $d=15 \mathrm{~mm}$ but it is not so important compared to simplification of the corrector ring fabrication. 

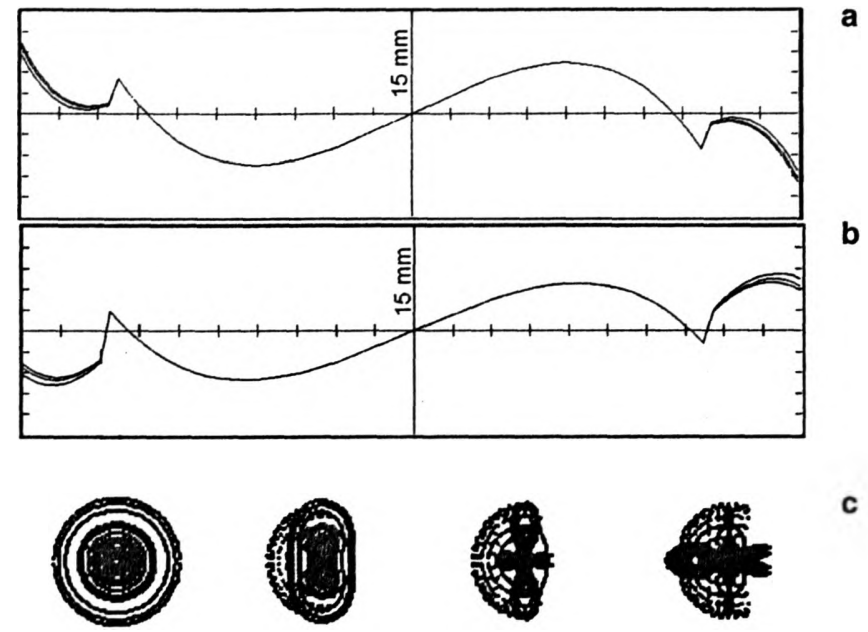

Fig. 9. FD system with "circular arc" substitution of the aspheric corrector ring profile: a - the transverse aberration for $0^{\circ}$ field of view, $\mathbf{b}$ - the transverse aberration for $20^{\circ}$ field of view, $\mathbf{c}$ - spot diagrams for $0^{\circ}, 10^{\circ}, 15^{\circ}, 20^{\circ}$ fields of view (from the left).

We can see the principle of the FD telescope correction (Fig. 9). There are the uncorrected primary spherical aberration for inner part of the system and the balanced oblique spherical aberration for the outer (annular) part of the system. There are three curves in the outer part which are given by refractive properties of the corrector ring. They show a residual spherochromatism in wavelength region between $300 \mathrm{~nm}$ and $400 \mathrm{~nm}$. It is a range where the nitrogen mostly emits fluorescence light in air shower. The spherochromatism is usually the limit aberration in the classical Schmidt camera but as you can see in the case of FD telescope system it is the irrelevant aberration. The problem is the choice of suitable material for the corrector ring. It is quite restricted in the given wavelength region.

\section{Real construction of the FD telescope}

The CAD drawing and the prototype photo are seen in Fig. 10. From the left to the right there are the circular diaphragm, the UV transmitting filter glass, the corrector ring (all of these near the radius of mirror curvature), the detector and the main spherical mirror. Various national participant groups made the individual optical elements of FD prototype.

The UV filter "window" is made from smaller rectangular tables of $3.25 \mathrm{~mm}$ thick UG6 Schott glass (Italian group). The corrector ring has outer diameter $2200 \mathrm{~mm}$ and the annular width $250 \mathrm{~mm}$. The maximum thickness is less than $12 \mathrm{~mm}$. The material of ring is UV acrylic (German group) or BK7 optical glass (Brazilian group). For practical reasons of fabrication, the ring is split into 24 pieces. The detector (the 

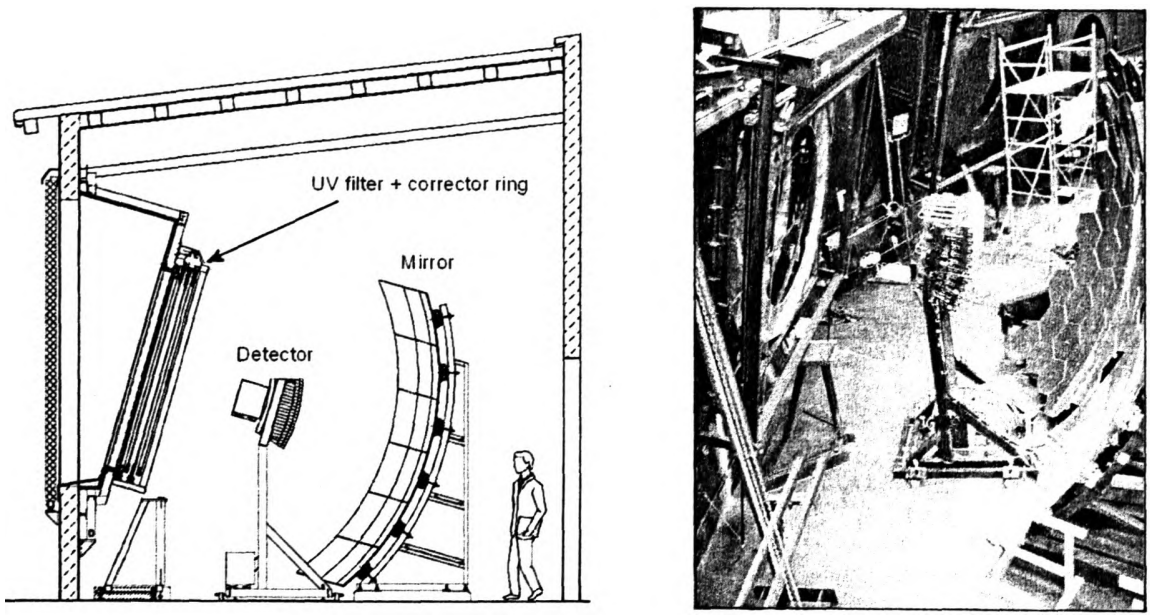

Fig. 10. CAD drawing and photo of the FD prototype.

camera) of the FD telescope is an array of 440 hexagonal photomultipliers, which are arranged on the curved focal surface. Camera size is $940 \times 860 \mathrm{~mm}$ (Italian group).

The main mirror is spherical with radius of curvature $R=3400 \mathrm{~mm}$. The shape of the mirror is nearly square due to the square field of view and the size is ca $3600 \times 3600 \mathrm{~mm}$. For practical reasons of total weight it is segmented into smaller "square" (German group) or hexagonal elements (Czech group).

The first FD prototype was installed in May 2001. The telescope is operated at moonless nights and the first images of the air showers have been recorded.

\section{Conclusions}

The Pierre Auger Observatory will study the highest known energy cosmic showers focusing attention on energies above $5 \times 10^{19} \mathrm{eV}$. The important part of this observatory is the fluorescence telescope. The unusual optical layout of the fast wide-angle telescope of the FD has been described with its basic optical quality properties.

Acknowledgments - We thank the Ministry of Education of the Czech Republic for its financial support under grants LNO0A006, Ingo LA 134 and the Grand Agency of Academy of Sciences of the Czech Republic for project A1010928.

\section{References}

[1] The Pierre Auger Observatory - Design Report, 1997

[2] Hrabovsky M., Palatka M., Schovanek P., Grygar J., Ridky J., Soukup L., The optical analysis of the proposed Schmidt camera design, Auger technical note, GAP-99-25: www.auger.org/admin-cgi-bin-woda-gap_notes.pl. 
[3] Linfoot E.H., Recent Advances in Optics, Clarendon Press, Oxford 1955, pp. 202-208.

[4] Palatka M., Hrabovsky M., Schovanek P., Ridky J., Grygar J., Soukup L., Bifocal Optical System of the Schmidt Camera (Design of the Corrector Ring), Auger technical note, GAP-2000-002: www.auger.org/admin-cgi-bin-woda-gap_notes.pl.

[5] Palatka M., Hrabovsky M., Schovanek P., Ridky J., Grygar J., Soukup L., Analysis of Possible Substitution of the Aspheric Surface of the Corrector Annulus by the Shape with Simpler Production Technology, Auger technical note, GAP-2000-003: www.auger.org/admin-cgi-bin-woda-gap_notes.pl

[6] SIGMA 2100, Optical Design Software, KIDGER OPTICS.

Received Septemver 19, 2002

in revised form December 2, 2002 